\title{
BMJ Open Consensus validation of the POSAMINO (POtentially Serious Alcohol-Medication INteractions in Older adults) criteria
}

\author{
Alice E Holton, ${ }^{1}$ Paul J Gallagher, ${ }^{1}$ Cristín Ryan, ${ }^{1}$ Tom Fahey, ${ }^{2}$ Gráinne Cousins ${ }^{1}$
}

To cite: Holton $\mathrm{AE}$, Gallagher PJ, Ryan $\mathrm{C}$, et al. Consensus validation of the POSAMINO (POtentially Serious AlcoholMedication INteractions in Older adults) criteria. BMJ Open 2017;7:e017453. doi:10.1136/ bmjopen-2017-017453

- Prepublication history and additional material for this paper are available online. To view these files, please visit the journal online (http://dx.doi. org/10.1136/bmjopen-2017017453).

Received 23 April 2017

Revised 6 September 2017

Accepted 11 September 2017

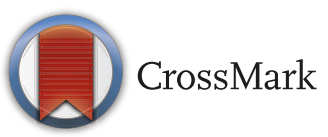

${ }^{1}$ School of Pharmacy, Royal College of Surgeons in Ireland (RCSI), Dublin 2, Ireland ${ }^{2}$ Division of Population Health Science, HRB Centre for Primary Care Research, Royal College of Surgeons in Ireland (RCSI), Dublin 2, Ireland

Correspondence to

Alice E Holton;

aliceholton@rcsi.ie

\section{ABSTRACT}

Objectives Older adults are particularly vulnerable to adverse effects from concurrent alcohol and medication use. However, there is limited evidence regarding the prevalence of these adverse outcomes among older adults, and there is a lack of consensus regarding what constitutes an alcohol-interactive medicine. The objective of this study was to develop an explicit list of potentially serious alcoholmedication interactions for use in older adults.

Design Following a systematic review, review of drug compendia and clinical guidance documents, a two-round Delphi consensus method was conducted.

Setting Ireland and the United Kingdom (UK), primary care and hospital setting.

Participants The Project Steering Group developed a list of potentially serious alcohol-medication interactions. The Delphi panel consisted of 19 healthcare professionals (general practitioners, geriatricians, hospital and community pharmacists, clinical pharmacologists and pharmacists, and physicians specialising in substance misuse).

Results An inventory of 52 potentially serious alcoholmedication interactions was developed by the Project Steering Group. British National Formulary black dot warnings $(n=8)$ were included in the final criteria as they represent 'potentially serious' interactions. The remaining 44 criteria underwent a two-round Delphi process. In the first round, 13 criteria were accepted into the POtentially Serious Alcohol-Medication INteractions in Older adults (POSAMINO) criteria. Consensus was not reached on the remaining 31 criteria; 9 were removed and 8 additional criteria were included following a review of panellist comments. The remaining 30 criteria went to round 2 , with 17 criteria reaching consensus, providing a final list of 38 potentially serious alcohol-medication interactions: central nervous system $(n=15)$, cardiovascular system $(n=9)$, endocrine system $(n=5)$, musculoskeletal system $(n=3)$, infections $(n=3)$, malignant disease and immunosuppression ( $n=2)$, and respiratory system $(n=1)$. Conclusions POSAMINO is the first set of explicit potentially serious alcohol-medication interactions for use in older adults. Following future validation studies, these criteria may allow for the risk stratification of older adults at the point of prescribing.

\section{BACKGROUND}

Population demographics are changing globally, with the proportion and age of the older
Strengths and limitations of this study

- The POtentially Serious Alcohol-Medication INteractions in Older adults criteria were developed in a robust fashion, using a two-step process involving a systematic review and two-round Delphi process.

- The Delphi consensus technique used in this study is flexible and enabled communication from a diverse group of healthcare professionals from both the UK and Ireland.

- The final criteria will need to be further validated prospectively to quantify the magnitude of risk posed for each criterion for adverse outcomes in older adults.

- Once validated, these criteria have the potential to inform medical decision making and enable healthcare professionals to risk stratify older adults at the point of prescribing and prioritise alcohol screening and brief interventions in high-risk groups.

population continuing to increase. ${ }^{1}$ While alcohol consumption tends to decline with age, older adults are inclined to drink more frequently. ${ }^{2}$ Ageing is associated with a variety of physiological changes, which may place older adults at an increased risk of alcohol-related health problems. ${ }^{34}$ In fact, compared with younger adults, they encounter a disproportionate burden of alcohol-related harm, with reported alcohol-related deaths highest among those aged 55 to 74 years in England in $2010{ }^{4}$

Polypharmacy is also increasing in older adults ${ }^{5}$ and certain medicines may interact with alcohol, increasing the risk of adverse events such as sedation, hypotension, gastrointestinal bleeds, hypoglycaemia and liver damage ${ }^{67}$ In a previous observational study, moderate alcohol consumption increased the risk of adverse drug reactions by $24 \%$ among older adults. ${ }^{8}$ Alcohol interactive (AI) medicines may interact with alcohol by altering the effects (pharmacodynamic) or 
metabolism (pharmacokinetic) of the medication and/ or alcohol. ${ }^{7}$ The interactions may occur with any alcohol or may follow a dose response, with the severity and risk of interactions increasing with increasing levels of alcohol consumption. ${ }^{6}$

While a number of studies have investigated the concurrent use of alcohol and AI medicines among older adults, ${ }^{9}$ there is still a lack of agreement regarding the inclusion of AI medicines across studies. Several studies have reported on a broad range of medication classes, using different drug reference sources and software to identify medications as AI, thus leading to a lack of consistency in the inclusion of these medicines. ${ }^{9}$ Other studies focused on psychotropic medications alone. ${ }^{9}$

Despite the findings of our previous systematic review, highlighting a high prevalence of concurrent use among older adults, no study to date has examined longitudinal associations of concurrent use with adverse outcomes. ${ }^{9} \mathrm{An}$ evidence-based list of potentially serious alcohol-medicine interactions for older adults has potential in a clinical setting, once validated, allowing for the identification of older adults whose alcohol consumption places them at increased risk and who would benefit from a preventative intervention. Therefore, the aim of this study is to derive the first set of explicit potentially serious alcoholmedication interactions in older adults.

\section{METHODS}

\section{Study design}

A Delphi consensus technique was used to develop the list of potentially serious alcohol-medication interactions in older adults. The Delphi method allows a consensus opinion to be reached among a panel of experts through an iterative process of questionnaires. ${ }^{10}$ Ethics approval for this study was obtained from the Royal College of Surgeons in Ireland (RCSI) (reference number REC 1097). A Project Steering Group comprising academic and clinical pharmacists, a general practitioner and an epidemiologist (authors) from the RCSI School of Pharmacy and Health Research Board Primary Care Centre was formed to develop the initial list of potentially serious alcohol-medication interactions and to later oversee the Delphi consensus study.

\section{Compilation of initial list of potentially serious alcohol- medication interactions}

Following a comprehensive systematic search using MEDLINE (PubMed), Embase, Scopus and Web of Science databases, ${ }^{9}$ an extensive list of medications with potential to interact with alcohol was identified by the Project Steering Group. A combination of the following keywords and MeSH terms were used: 'ethanol', 'alcohol', 'drug interactions', 'drug alcohol interaction' and 'aged'. This search was supplemented by a search in Google Scholar and by hand searching references of retrieved articles. The search was restricted to English-language articles and articles published since January 1990.
Furthermore, the British National Formulary (BNF), Stockley's Drug Interactions and Martindale Complete Drug Reference drug compendia were also searched. ${ }^{11-13}$ Additional documents such as clinical guidance documents ${ }^{14}$ and previous reviews ${ }^{6} 7$ were also accessed. Information extracted included medication name/class, potential adverse outcome(s), whether an interaction is likely to occur with any alcohol consumption or with heavy consumption using national low risk drinking guidelines ${ }^{1516}$ and, if reported, evidence supporting the interaction. The list of medications was organised according to the BNF physiological classification system. There was considerable heterogeneity across reference sources in terms of identifying medications as having potential to interact with alcohol, with no age-specific information for interactions. Furthermore, there were inconsistencies in relation to the quantity of concurrent alcohol consumption that should be avoided. For example, the Martindale mentioned that alcohol combined with non-steroidal anti-inflammatory drugs (NSAIDs) may increase the risk of gastrointestinal bleeds with no mention of alcohol consumption patterns, Stockley's described that both NSAIDs and excessive alcohol use carry the risk of gastrointestinal adverse effects, while NSAIDs and alcohol were not included as an interaction in the BNF. For the next step, the Project Steering Group reviewed and assessed each medication or drug class. Using the definition of 'necessary to avoid' previously described by Dreischulte et $a l,{ }^{17}$ it was considered necessary to avoid concurrent alcohol with a specific medication if, in an average older adult, concurrent use of alcohol with this medication would be considered unsafe because (a) there is sufficient evidence that the patient is likely to be harmed and (b) the likely harm to the patient is large enough to be clinically significant.

Drugs were excluded for the following reasons: their interaction with alcohol was not considered serious, that is, unlikely to cause significant harm to the patient (eg, selective serotonin reuptake inhibitors and alcohol) ${ }^{13}$; the medication is not licensed for use in older adults; the medication is only administered in a hospital environment (eg, trabectedin); and interaction with alcohol may only occur during alcohol withdrawal or has been withdrawn from the market in the UK or Ireland. Following these exclusions, a truncated list was further reviewed by the Project Steering Group by a consensus discussion. In this process, the Project Steering Group agreed that BNF black dot warnings would be included in the final list. BNF black dots refer to potentially serious drug-alcohol interactions where concurrent use should be avoided or only undertaken with caution and appropriate management. In the paper version of the BNF, these potentially serious drug-alcohol interactions are highlighted to prescribers by flagging them with a 'black dot'. ${ }^{11}$ Furthermore, the amount of alcohol to be avoided with each medication class was defined through group discussion, based on both the evidence available and the members' own clinical experience. Additionally, it was agreed that the following 
adverse outcomes associated with concurrent use were defined using clinical guidance documents and reviews to ensure clarity among all participants: orthostatic hypotension, ${ }^{18}$ hypoglycaemia ${ }^{19}$ and lactic acidosis. ${ }^{20}$

\section{Selection of the Delphi panel}

A total of 51 experts from Ireland and the UK were invited (via email or letter) to participate as part of the Delphi consensus panel. The experts invited to participate on the Delphi consensus panel were peer recognised by the Project Steering Group or nominated by other panel members on the basis of their clinical experience or knowledge of alcohol-medication interactions or care of older persons.

Reasons for non-participation were not required; however, in some instances they were provided, which included time commitments and lack of clinical knowledge of alcohol-medication interactions. In total, 19 participants $(37 \%)$ agreed to participate and written consent was received from all participants before the study. The panel consisted of general practitioners (GPs) $(n=5)$, geriatricians $(n=3)$, hospital pharmacists with expertise in care of older adults $(n=3)$, community pharmacists $(n=3)$, clinical pharmacologists $(n=2)$, clinical pharmacists specialising in substance misuse $(n=2)$ and a physician specialising in substance misuse $(n=1)$. Panel members were not provided with compensation for participation.

\section{The Delphi validation technique and process}

An online questionnaire was piloted by two pharmacists and two GPs, to identify any potential problems and to approximate the completion time for the survey. Following amendments, the 19 participants were sent a link to the online POtentially Serious Alcohol-Medication INteractions in Older adults (POSAMINO) questionnaire (via SurveyGizmo) in March 2016. Participants were given 4 weeks to complete the online questionnaire and all participants were sent a reminder email after 2 weeks to encourage participation. The questionnaire was both anonymous and confidential.

The quantity of alcohol per standard drink (SD) (10 g alcohol in Ireland) or unit ( $8 \mathrm{~g}$ alcohol in the UK) was defined at the beginning of the survey. As some interactions are listed with any alcohol consumption and others with 'heavy' consumption, we provided definitions of both at the beginning of the questionnaire. Any alcohol consumption was defined as $\geq 1 \mathrm{SD}$ of alcohol in Ireland or $\geq 1$ unit of alcohol in the UK, with heavy consumption defined according to Irish and UK national low risk drinking guidelines. ${ }^{15} 16$ The panel were instructed to evaluate each potential interaction listed according to the 'necessary to avoid' framework described above. ${ }^{17}$ Medications were categorised according to the BNF physiological classification system. Each statement was presented in the same format stating: It is necessary to avoid (quantity of alcohol) (any or heavy) with (medications/drug class), followed by a brief rationale for the statement. For example: It is necessary to avoid: Any alcohol consumption with first-generation antihistamines (for example promethazine). (Rationale: Concurrent alcohol consumption with first-generation antihistamines can increase the risk of sedation).

Participants were asked to rate their agreement with each statement, using a five-point Likert scale (1=strongly disagree to $5=$ strongly agree), along with any additional comments or suggestions for additional medicines to be included.

After each round, the median and IQRs were calculated for each statement. Consistent with previous Delphi consensus studies, the consensus level required for a statement to be retained was defined a priori as a median of 4 or 5 with a lower quartile value of $\geq 4 .{ }^{21}$ If a statement had an upper quartile value of $\leq 2$, this indicated there was general disagreement with the statement between panel members, and the statement was rejected. If group consensus was not reached, the criteria were reviewed by the Project Steering Group and were removed based on comments or revised and included in the second questionnaire. In the second questionnaire, panellists were provided with links to the most recent evidence relating to each of the alcohol-medication interactions, to help facilitate their decisions due to uncertainty in round 1 . As with round 1 , the median response and IQR were calculated and evaluated by the Project Steering Group using the same thresholds to determine consensus between the panel members. If consensus was not reached following the second round, the statement was rejected. Statistical analysis was performed using STATA V.13 and Microsoft Excel 2010.

\section{RESULTS}

Following an initial review of the literature, Anatomical Therapeutic Chemical codes for a total of $364 \mathrm{AI}$ medicines were extracted by the Project Steering Group (online supplementary file 1). Medicines were classified according to drug classes and organised according to BNF physiological classification systems. From this list, a total of 63 statements were initially compiled based on the 'necessary to avoid' framework. ${ }^{17}$ Following group discussions, 11 statements were removed/updated or merged together, based on the steering group's clinical knowledge or experience with medicines. For example, the steering group decided to no longer classify benzodiazepines according to the duration of action, as both long acting and short acting have the potential to interact with alcohol. Furthermore, a total of eight BNF black dot warnings were included in the final criteria as they represent potentially serious interactions.

All 19 panel members completed the round 1 questionnaire in which 44 statements were presented. Consensus was reached on 13 statements, with no statements rejected (table 1). Thirty-one statements were reviewed by the steering group, with nine statements removed based on comments from the panel, if the interaction was not relevant to older adults or the interaction was not of clinical 


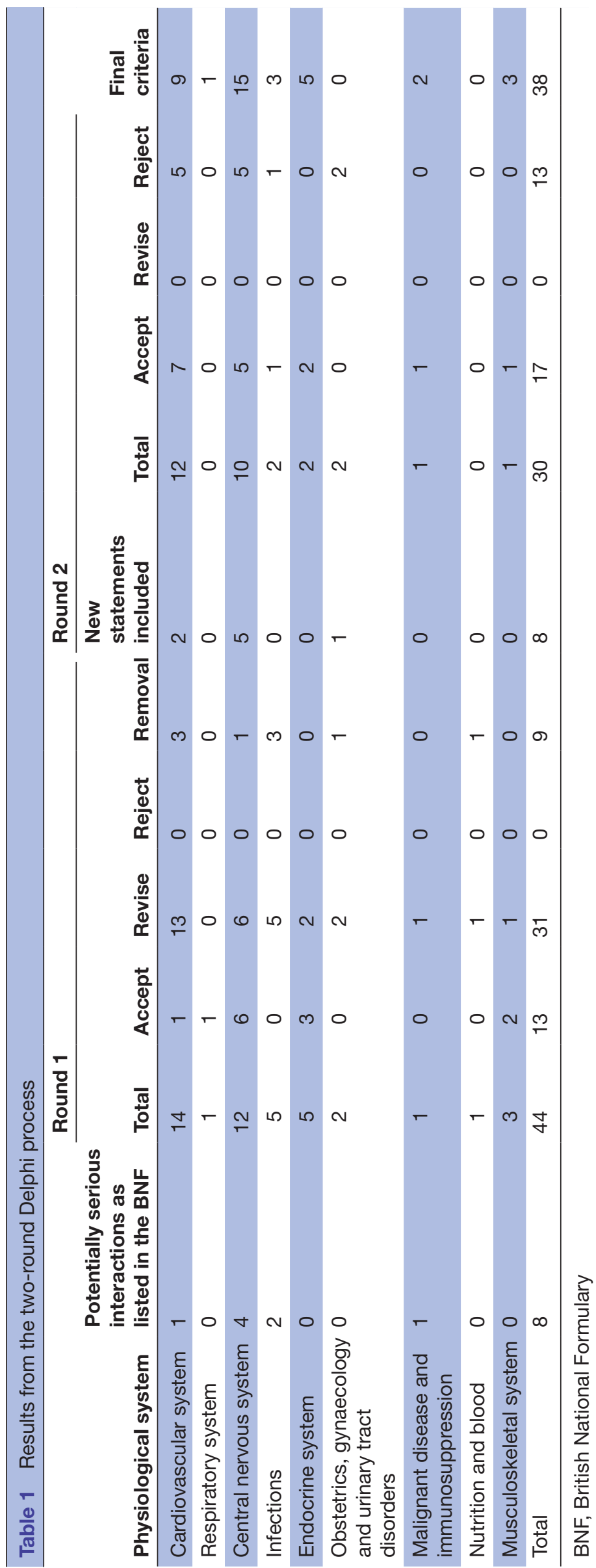


significance, for example, heavy alcohol consumption with vitamin A preparations. Furthermore, eight new statements were included in round 2 based on comments from panellists at the first round.

A total of 18 out of the 19 recruited participants completed the round 2 questionnaire. Of the 30 statements included in the second questionnaire, consensus was reached for 17 statements. The remaining 13 statements were rejected as no consensus was reached. In total, consensus was reached for 30 potentially serious drug-alcohol interactions in older adults, and with the inclusion of BNF black dot warnings, the final list was 38 statements.

This final 38-item POSAMINO criteria (see box 1) were organised over the following physiological systems: central nervous system $(\mathrm{n}=15)$, cardiovascular system $(n=9)$, endocrine system $(n=5)$, musculoskeletal system $(n=3)$, infections $(n=3)$, malignant disease and immunosuppression $(\mathrm{n}=2)$ and respiratory system $(\mathrm{n}=1)$.

\section{DISCUSSION}

\section{Principal findings in relation to previous studies}

While older adults experience a disproportionate burden of alcohol-related harm, ${ }^{4}$ research suggests that healthcare professionals are less likely to discuss alcohol consumption with older adults. ${ }^{22}{ }^{23}$ Flagging older adults at the point of prescribing an AI medication may facilitate targeted screening and interventions to help reduce harm. Despite the high propensity for alcohol-medication interactions among community-dwelling older adults, our recent systematic review has highlighted that there is still a lack of consensus regarding what constitutes an AI medication. ${ }^{9}$ This study reports the development of a set of 38 explicit criteria, POSAMINO, for identifying older adults at risk of potentially serious alcohol-medication interactions.

The final POSAMINO criteria consist of seven different drug classes, with central nervous system (CNS) medicines representing $40 \%$ of the criteria. Estimates from our previous study indicate that approximately one in five older Irish adults are exposed to CNS agents, with over half of patients using CNS agents also reporting concurrent alcohol consumption. ${ }^{24}$ Nine of the final 38 criteria concern cardiovascular agents, another widely used drug class among aged adults. ${ }^{24}$ There is also a high risk of adverse effects associated with these agents, with antiplatelets, diuretics and anticoagulants identified as the most common drug classes involved in preventable drug-related admissions, in a previous study. ${ }^{25}$ Analgesics such as non-steroidal anti-inflammatory drugs (NSAIDs), opioids, paracetamol and gabapentin (used for neuropathic pain) were also included in the final POSAMINO criteria. The prevalence of chronic pain increases with age and older adults may consume alcohol to help cope and manage their pain. ${ }^{26}$ This behaviour may also predispose older adults to adverse outcomes associated with concurrent use with analgesics (for example increased sedation, falls and gastrointestinal bleeds).
The development of the POSAMINO criteria is important for several reasons. In the absence of an explicit list of AI medications, multiple drug reference sources have been used in previous studies leading to a lack of consistency in the inclusion of medications across studies, ${ }^{24}$ 27-30 which may result in biased estimates of potential risk among older adults. Furthermore, some of these interactions may be theoretical with trivial clinical significance. Therefore, this study developed a consensus-based set of explicit criteria for potentially serious alcohol-medication interactions in older adults rather than an exhaustive list of medications with potential to interact with alcohol. Consistent with existing literature, the POSAMINO criteria classify CNS agents as AI medications with the potential to cause serious harm to older adults. $^{82427-41}$ However, some of the previous studies focused on sedatives/hypnotics only. ${ }^{33} 3941$ We identified a number of additional CNS agents, such as anti-Parkinson's drugs (eg, pramipexole, apomorphine and levodopa), which may be overlooked if we focus solely on sedatives or hypnotics.

Furthermore, the POSAMINO criteria also includes a wide range of other drug classes, such as cardiovascular, respiratory system, musculoskeletal, malignant disease, infections or endocrine agents that were not all considered among previous studies. ${ }^{82728}{ }^{30-32} 35$ It is also important to note that there has been little emphasis on the adverse outcomes or severity of these potential interactions. ${ }^{9} 29$ The development of the POSAMINO criteria may help identify older adults at risk of potentially serious alcohol-medicine interactions in the future.

\section{Clinical implications and future research}

This study adds to a growing body of research investigating the concurrent use of alcohol and AI medicines in older adults. Undoubtedly, adverse drug events (ADEs) represent a major burden on healthcare, with ADEs detected in $26.3 \%$ of patients aged $\geq 65$ years admitted to an Irish hospital with acute illnesses. ${ }^{42}$ The increasing use of multiple medications, ${ }^{5}$ combined with an increased frequency of alcohol consumption ${ }^{2}$ and age-related physiological changes, may predispose older adults to these adverse events. The significant burden of alcohol-related harm and mortality among older adults ${ }^{4}$ indicates a pressing need for future interventions to minimise risk. However, prior to informing clinical or public health initiatives, the final criteria developed from this study will require further validation to prospectively quantify the magnitude of risk posed for each criterion for adverse outcomes in older adults. In particular, the quantity of alcohol specified in each criterion will need to be further evaluated in prospective studies. Once validated, these criteria have the potential to inform medical decision making and enable healthcare professionals to risk stratify older adults at the point of prescribing and prioritise alcohol screening and brief interventions in high-risk groups. This is essential, as older people have previously reported little knowledge 
Box 1 Final POtentially Serious Alcohol-Medication INteractions in Older adults criteria $(n=38)$

\section{Cardiovascular system}

1. Heavy alcohol consumption with multiple antihypertensive combinations

Rationale: Concurrent use of alcohol consumption and antihypertensives may increase the risk of orthostatic hypotension.

2. Heavy alcohol consumption with warfarin (and phenindione)

Rationale: Heavy episodic alcohol consumption is associated with an increased risk of major bleeds.

3. Heavy alcohol consumption with regular use of low-dose aspirin $(75 \mathrm{mg})$

Rationale: Heavy alcohol consumption combined with aspirin may cause a small increase in gastrointestinal blood loss.

4. Heavy alcohol consumption with both regular and as required nitrates (eg, glyceryl trinitrate, isosorbide dinitrate and isosorbide mononitrate)

Rationale: The combined haemodynamic effects of alcohol and nitrates may increase the risk of exaggerated hypotension.

5. Heavy alcohol consumption with the vasodilatory medication nicorandil

Rationale: The combined haemodynamic effects of alcohol and nicorandil may increase the risk of exaggerated hypotension.

6. Heavy alcohol consumption with the combined use of both nitrates and vasodilator medication (eg, nicorandil)

Rationale: The combined haemodynamic effects of alcohol with nitrates and vasodilator drugs may increase the risk of exaggerated

hypotension.

7. Heavy alcohol consumption with diuretics (eg, loop diuretics (furosemide), thiazide diuretics (bendroflumethiazide) and potassium-sparing diuretics (amiloride))

Rationale: The concurrent use of alcohol and antihypertensives may increase the risk of orthostatic hypotension.

8. Heavy alcohol consumption with alpha blockers (eg, terazosin)

Rationale: The concurrent use of alcohol and antihypertensives may increase the risk of orthostatic hypotension.

9. Heavy alcohol consumption with centrally acting antihypertensives (eg, clonidine or methyldopa)

Rationale: Alcohol consumption combined with centrally acting antihypertensives may increase the risk of sedation and/or orthostatic hypotension.

Respiratory system

1. Any alcohol consumption with first-generation antihistamines (eg, promethazine)

Rationale: Concurrent alcohol consumption with first-generation antihistamines can increase the risk of sedation.

Central nervous system (CNS)

1. Heavy alcohol consumption with benzodiazepines (eg, diazepam) and benzodiazepine-related medications (eg, zopiclone)

Rationale: Alcohol consumption combined with benzodiazepines and benzodiazepine-related medications may enhance CNS depressant effects.

2. Heavy alcohol consumption combined with opioids

Rationale: Alcohol consumption combined with opioids may enhance CNS depressant effects of alcohol.

3. Heavy alcohol consumption with duloxetine

Rationale: Heavy alcohol consumption combined with duloxetine may increase the risk of hepatotoxicity.

4. Heavy alcohol consumption with all antipsychotics

Rationale: Alcohol consumption combined with antipsychotics may increase the risk of sedation.

5. Any alcohol consumption with barbiturates

Rationale: Alcohol consumption combined with barbiturates may increase the risk of sedation.

6. Heavy alcohol consumption with antiepileptic drugs (AEDs)

Rationale: Heavy alcohol consumption can increase the risk of seizures and sedation in patients taking AEDs.

7. Any alcohol consumption with tricyclic antidepressants

Rationale: Alcohol consumption combined with antidepressants may enhance the CNS depressant effects of alcohol.

8. Any alcohol consumption with tetracyclic antidepressants

Rationale: Alcohol consumption combined with antidepressants may enhance the CNS depressant effects of alcohol.

9. Any alcohol consumption with mirtazapine

Rationale: Alcohol consumption combined with antidepressants may enhance the CNS depressant effects of alcohol.

10. Any alcohol consumption with monoamine oxidase inhibitors (MAOIs)

Rationale: A potentially life-threatening hypertensive reaction can develop in patients taking non-selective MAOls who consume drinks rich in tyramine (eg, wines, beers and non-alcoholic beers)

11. Heavy alcohol consumption with long-term regular paracetamol use (eg, $1 \mathrm{~g}$ four times a day)

Rationale: Heavy alcohol consumption may increase the risk of paracetamol hepatotoxicity especially if alcohol intake is abruptly stopped.

12. Heavy alcohol consumption with gabapentin (when used for neuropathic pain)

Rationale: Alcohol combined with medications for neuropathic pain may increase the risk of sedation.

13. Heavy alcohol consumption with pramipexole or amantadine

Rationale: Alcohol combined with pramipexole or amantadine may increase the risk of sedation

14. Heavy alcohol consumption with apomorphine

Rationale: Alcohol combined with apomorphine may increase the risk of orthostatic hypotension.

15. Heavy alcohol consumption with levodopa (alone or in combination with carbidopa)

Rationale:Alcohol combined with levodopa (alone or in combination with carbidopa) may increase the risk of orthostatic hypotension.

\section{Endocrine}

1. Heavy alcohol consumption with insulin

Rationale: Alcohol consumption may enhance the hypoglycaemic effect of insulin. 


\section{Box 1 Continued}

2. Heavy alcohol consumption with metformin

Rationale: Heavy alcohol consumption combined with metformin may increase the risk of lactic acidosis.

3. Heavy alcohol consumption with sulphonylureas

Rationale: Alcohol consumption can enhance the hypoglycaemic effects of antidiabetics.

4. Heavy alcohol consumption with meglitinides (eg, nateglinide)

Rationale: Alcohol consumption can enhance the hypoglycaemic effects of antidiabetics.

5. Heavy alcohol consumption with thiazolidinediones (eg, pioglitazone)

Rationale: Alcohol consumption can enhance the hypoglycaemic effects of antidiabetics.

Musculoskeletal and joint diseases

1. Heavy alcohol consumption with any non-steroidal anti-inflammatory drugs (NSAIDs) (including COX-2 inhibitors)

Rationale: Heavy alcohol consumption and NSAID use carry an increased risk of gastrointestinal bleeds.

2. Heavy alcohol consumption combined with methotrexate or leflunomide

Rationale: Heavy alcohol consumption combined with methotrexate or leflunomide may increase the risk of hepatotoxicity.

3. Heavy alcohol consumption with oral muscle relaxants (eg, baclofen)

Rationale: Concurrent alcohol consumption and muscle relaxants can increase the risk of CNS depression.

Malignant disease and immunosuppression

1. Any alcohol consumption with procarbazine

Rationale: A disulfiram-like reaction can occur when alcohol is given with procarbazine.

2. Heavy alcohol consumption with interferon alpha or interferon beta

Rationale: Heavy alcohol consumption combined with interferons may increase the risk of hepatotoxicity and reduce the response to treatment with interferon.

Infections

1. Heavy alcohol consumption with antimycobacterial medications such as isoniazid, pyrazinamide, ethionamide and rifampicin (alone or in combination)

Rationale: Alcohol combined with antimycobacterial medications can increase the risk of hepatotoxicity.

2. Any alcohol consumption with cycloserine

Rationale: Alcohol consumption may increase the risk of seizures in patients taking cycloserine.

3. Any alcohol consumption with metronidazole or tinidazole

Rationale: A disulfiram-like reaction can occur when alcohol is given with metronidazole.

about risks associated with the concurrent use of alcohol and medications. ${ }^{43}$

\section{Strengths and limitations}

The criteria were developed in a robust fashion, using a two-step process involving a systematic review and two-round Delphi process. The Delphi technique is flexible and for this study enabled communication from a group of 19 healthcare professionals from both the United Kingdom and Ireland. Eighteen of the 19 participants completed the two rounds, with participants providing numerous comments and suggestions in both rounds. Similar to previous studies, participants remained anonymous and were not provided with feedback following the first round in order to remove the risk of potential bias. ${ }^{21}$

Inevitably, there were limitations to this Delphi study. With all Delphi studies, participants' judgement may be subjective. In order to reduce this potential bias, a diverse group of healthcare professionals with expertise or interest in the care of older adults were selected. With explicit criteria, there is also a need for regular updates and revision due to the availability of more up-to-date information after development. ${ }^{44}$ The developed POSAMINO criteria do not apply to older adults diagnosed with an alcohol use disorder, as chronic heavy alcohol consumption can substantially increase the activity of the cytochrome P450 metabolising enzyme CYP2E1. ${ }^{7}$ Finally, older adults may also experience chronic illnesses that affect the metabolism of both alcohol and medications. As a result, it is important that healthcare professionals also consider these comorbid diseases when assessing the risk for potential adverse outcomes. ${ }^{45}$

\section{CONCLUSIONS}

Using a systematic review and a two-round Delphi consensus method, we have developed the first set of explicit potentially serious alcohol-medication interactions in older adults (POSAMINO). Following future validation studies, these criteria may allow for the risk stratification of older adults at the point of prescribing, and prioritise alcohol screening and brief alcohol interventions in high-risk groups.

\section{Twitter @alholtonkelly}

Acknowledgements We would like to thank all Delphi participants who contributed to the development of the POSAMINO criteria.

Contributors AEH, PJG, CR, TF and GC (the Project Steering Group) conceived and designed this study. AEH and GC conducted initial literature search. AEH communicated with Delphi participants. AEH analysed the data after each round. All Project Steering Group members were involved in interpretation of the data. AEH and GC drafted the manuscript. All coauthors revised the manuscript and gave the approval for publication.

Funding This study was funded by the Royal College of Surgeons in Ireland (RCSI), School of Pharmacy, Dublin 2, Ireland. 
Competing interests None declared.

Ethics approval Ethical approval for this study was approved by the Royal College of Surgeons in Ireland (RCSI) ethics committee (REC 1097).

Provenance and peer review Not commissioned; externally peer reviewed.

Data sharing statement Additional data are available by request from the corresponding author.

Open Access This is an Open Access article distributed in accordance with the Creative Commons Attribution Non Commercial (CC BY-NC 4.0) license, which permits others to distribute, remix, adapt, build upon this work non-commercially, and license their derivative works on different terms, provided the original work is properly cited and the use is non-commercial. See: http://creativecommons.org/ licenses/by-nc/4.0/

(c) Article author(s) (or their employer(s) unless otherwise stated in the text of the article) 2017. All rights reserved. No commercial use is permitted unless otherwise expressly granted.

\section{REFERENCES}

1. United Nations, Department of Economic and Social Affairs PD. World Population Prospects: the 2015 revision, key findings and advance tables. New York, 2015. https://esa.un.org/unpd/wpp/ Publications/Files/Key_Findings_WPP_2015.pdf

2. Britton A, Ben-Shlomo $Y$, Benzeval M, et al. Life course trajectories of alcohol consumption in the United Kingdom using longitudinal data from nine cohort studies. BMC Med 2015;13:47.

3. Huang AR, Mallet L, Rochefort CM, et al. Medication-related falls in the elderly: causative factors and preventive strategies. Drugs Aging 2012;29:359-76.

4. Wadd S, Papadopoulos C. Drinking behaviour and alcohol-related harm amongst older adults: analysis of existing UK datasets. BMC Res Notes 2014;7:741.

5. Moriarty F, Hardy C, Bennett K, et al. Trends and interaction of polypharmacy and potentially inappropriate prescribing in primary care over 15 years in Ireland: a repeated cross-sectional study. BMJ Open 2015;5:e008656

6. Moore AA, Whiteman EJ, Ward KT. Risks of combined alcohol/ medication use in older adults. Am J Geriatr Pharmacother 2007;5:64-74.

7. Weathermon R, Crabb DW. Alcohol and medication interactions. Alcohol Res Health 1999;23:40-54.

8. Onder G, Landi F, Della Vedova C, et al. Moderate alcohol consumption and adverse drug reactions among older adults. Pharmacoepidemiol Drug Saf 2002;11:385-92.

9. Holton AE, Gallagher P, Fahey T, et al. Concurrent use of alcohol interactive medications and alcohol in older adults: a systematic review of prevalence and associated adverse outcomes. BMC Geriatr 2017; $17: 148$

10. Hsu C-C, Sandford BA. The Delphi technique: making sense of consensus. Practical assessment, research \& evaluation;12:1-8.

11. Committee JF. The British National Formulary (BNF). 69th edn. London-British: Medical Association and Royal Pharmaceutical Society of Great Britain, 2015.

12. Sweetman S. Martindale: the complete drug reference London: Pharmaceutical Press. J Med Libr Assoc 2012;100:75-6 https://www. medicinescomplete.com/

13. Stockley's Drug Interactions [online] [Internet]. Pharmaceutical Press https://www.medicinescomplete.com/mc/stockley/current/

14. National Institute on Alcohol Abuse and Alcoholism (NIAAA) Harmful interactions mixing alcohol with medicines. 2014 https//pubs.niaaa. nih.gov/publications/medicine/Harmful_Interactions.pdf

15. Hope. A standard drink in Ireland: what strength? Health service executive-alcohol implementation group, 2009.

16. Department of Health January. UK Chief Medical Officers' Alcohol Guidelines Review Summary of the proposed new guidelines. 2016. https://www.gov.uk/government/uploads/system/uploads/ attachment_data/file/489795/summary.pdf

17. Dreischulte T, Grant AM, McCowan C, et al. Quality and safety of medication use in primary care: consensus validation of a new set of explicit medication assessment criteria and prioritisation of topics for improvement. BMC Clin Pharmacol 2012;12:5.

18. Lanier JB, Mote MB, Clay EC. Evaluation and management of orthostatic hypotension. Am Fam Physician 2011;84:527-36.

19. European Medicines Agency (EMA) Guideline on clinical investigation of medicinal products in the treatment or prevention of diabetes mellitus Committee for Medicinal Products for Human Use. 2012.
http://www.ema.europe.eu/docs/en_GB_document_library/ Scientific guideline/2012/06/WC500129256.pdf

20. Salpeter SR, Greyber E, Pasternak GA, et al. Risk of fatal and nonfatal lactic acidosis with metformin use in type 2 diabetes mellitus: systematic review and meta-analysis. Arch Intern Med 2003;163:2594-602.

21. Cooper JA, Ryan C, Smith SM, et al. The development of the PROMPT (PRescribing Optimally in Middle-aged People's Treatments) criteria. BMC Health Serv Res 2014;14:484.

22. Duru OK, Xu H, Tseng $\mathrm{CH}$, et al. Correlates of alcohol-related discussions between older adults and their physicians. J Am Geriatr Soc 2010;58:2369-74.

23. Kuerbis A, Sacco P, Blazer DG, et al. Substance abuse among older adults. Clin Geriatr Med 2014;30:629-54.

24. Cousins G, Galvin R, Flood M, et al. Potential for alcohol and drug interactions in older adults: evidence from the Irish longitudinal study on ageing. BMC Geriatr 2014;14:57.

25. Howard RL, Avery AJ, Slavenburg S, et al. Which drugs cause preventable admissions to hospital? A systematic review. $\mathrm{Br} J$ Clin Pharmacol 2007;63:136-47.

26. Ahangari A, Stewart Williams J, Myléus A. Pain and alcohol consumption among older adults: findings from the World Health Organization Study on global AGEing and adult health, Wave 1. Trop Med Int Health 2016;21:1282-92.

27. Breslow RA, Dong C, White A. Prevalence of alcohol-interactive prescription medication use among current drinkers: United States, 1999 to 2010. Alcohol Clin Exp Res 2015;39:371-9.

28. Immonen S, Valvanne J, Pitkälä KH. The prevalence of potential alcohol-drug interactions in older adults. Scand J Prim Health Care 2013;31:73-8.

29. Pringle KE, Ahern FM, Heller DA, et al. Potential for alcohol and prescription drug interactions in older people. J Am Geriatr Soc 2005;53:1930-6.

30. Qato DM, Manzoor BS, Lee TA. Drug-alcohol interactions in older U.S. adults. J Am Geriatr Soc 2015;63:2324-31.

31. Adams WL. Potential for adverse drug-alcohol interactions among retirement community residents. J Am Geriatr Soc 1995;43:1021-5

32. Aira M, Hartikainen S, Sulkava R. Community prevalence of alcohol use and concomitant use of medication - a source of possible risk in the elderly aged 75 and older? Int $J$ Geriatr Psychiatry 2005;20:680-5

33. Del Río MC, Prada C, Alvarez FJ. Do Spanish patients drink alcohol while undergoing treatment with benzodiazepines? Alcohol 2002;26:31-4.

34. Du Y, Scheidt-Nave C, Knopf H. Use of psychotropic drugs and alcohol among non-institutionalised elderly adults in Germany. Pharmacopsychiatry 2008;41:242-51.

35. Forster LE, Pollow R, Stoller EP. Alcohol use and potential risk for alcohol-related adverse drug reactions among community-based elderly. J Community Health 1993;18:225-39.

36. Ilomäki J, Gnjidic D, Hilmer SN, et al. Psychotropic drug use and alcohol drinking in community-dwelling older Australian men: the CHAMP study. Drug Alcohol Rev 2013;32:218-22.

37. Ilomäki J, Korhonen MJ, Enlund $\mathrm{H}$, et al. Risk drinking behavior among psychotropic drug users in an aging Finnish population: the FinDrink study. Alcohol 2008;42:261-7.

38. John U, Baumeister SE, Völzke H, et al. Sedative, hypnotic, anxiolytic and opioid medicament use and its co-occurrence with tobacco smoking and alcohol risk drinking in a community sample. BMC Public Health 2007;7:337.

39. Lagnaoui R, Moore N, Dartigues JF, et al. Benzodiazepine use and wine consumption in the French elderly. $\mathrm{Br} J$ Clin Pharmacol 2001;52:455-6.

40. Sheahan SL, Coons SJ, Robbins CA, et al. Alcohol-use, and falls among older adults. J Behav Med 1995;18:127-40.

41. Veldhuizen S, Wade TJ, Cairney J. Alcohol consumption among Canadians taking benzodiazepines and related drugs. Pharmacoepidemiol Drug Saf 2009;18:203-10.

42. Hamilton $\mathrm{H}$, Gallagher P, Ryan $\mathrm{C}$, et al. Potentially inappropriate medications defined by STOPP criteria and the risk of adverse drug events in older hospitalized patients. Arch Intern Med 2011;171:1013-9.

43. Zanjani F, Hoogland Al, Downer BG. Alcohol and prescription drug safety in older adults. Drug Healthc Patient Saf 2013;5:13-27.

44. O'Mahony D, O'Sullivan D, Byrne S, et al. STOPP/START criteria for potentially inappropriate prescribing in older people: version 2. Age Ageing 2015;44:213-8.

45. Slattum PW, Hassan OE. et a/Medications, alcohol, and aging. In: Kuerbis A, Moore AA, Sacco P, Zanjani F, . eds. Alcohol and aging: clinical and public health perspectives. Cham: Springer International Publishing, 2016:117-29. p. 